\title{
Correlação entre as Concentrações Plasmáticas e Foliculares de IGF-I
}

Tese de Mestrado apresentada junto ao Departamento de Ginecologia e Obstetrícia da Faculdade de Medicina de Ribeirão Preto da Universidade de São Paulo em 12 de Setembro de 1997.

Autor: Dr. Gustavo Salata Romão

Orientador: Prof. Dr. Rui Alberto Ferriani

O conhecimento acerca dos fatores de crescimento semelhantes à insulina (IGFs) tem despertado interesse de muitos autores, haja visto a importância do seu papel modulador em diversas interações endócrinas e metabólicas no organismo. Em nível ovariano o IGF-I parece modular a atividade das gonadotrofinas sobre as células foliculares, sendo encontrado em grandes concentrações no fluido folicular. Entretanto a verdadeira origem do IGF-I folicular é assunto controverso.

No presente estudo foram comparadas as concentrações séricas e foliculares de IGF-I de vinte e duas pacientes submetidas a fertilização in vitro no Hospital das Clínicas de Ribeirão Preto para tratamento da infertilidade conjugal. A indução da ovulação foi realizada de acordo com os protocolos do serviço. As amostras de sangue foram obtidas na ocasião de administração de HCG e no dia da captação de oócitos; as amostras de fluido folicular foram obtidas durante a captação de oócitos.

Os resultados mostraram uma correlação significativa entre as concentrações de IGF-I no plasma e no fluido folicular, com tendência à diminuição dessas concentrações no fluido folicular em relação ao plasma.

Esses achados sugerem que a maior parte do IGFI folicular seja difundido a partir do plasma e que a produção local seja de importância secundária. Provavelmente a difusão do IGF-I seja facilitada durante a fase periovulatória quando ocorre aumento da vascularização e da permeabilidade vascular em torno do folículo dominante. Estas observações corroboram estudos recentes e contribuem para a melhor compreensão da influência dos fatores de crescimento no processo ovulatório e na fisiopatologia das anovulações crônicas.

Palavras-chave: Infertilidade. Fertilização in vitro. Indução da ovulação.

\section{Quimioterapia Primária em Câncer de Mama Localmente Avançado. Estudo Comparativo entre Dois Esquemas Terapêuticos com Intensificação de Doses}

Tese apresentada à banca examinadora do Instituto de Ginecologia da Universidade Federal do Rio de Janeiro como parte dos requisitos à obtenção do grau de Doutor em Medicina, Rio de Janeiro-RJ, em 06/05/97.

Autor: Dr. Maurício Augusto Silva Magalhães Costa

Orientador: Prof. Dr. Jacir Luiz Balen

Realizou-se estudo clínico prospectivo, fase III, multicêntrico, aberto, aleatório e comparativo. Foram avaliadas 60 pacientes portadoras de câncer de mama localmente avançado, estádio IIIA, divididas em dois grupos, que foram submetidas à quimioterapia primária, com ou sem intensificação de dose, por quatro ciclos, e à cirurgia.

Utilizou-se protocolo FEC 50 no grupo A (5-FU $500 \mathrm{mg} / \mathrm{m}^{2}$, epirrubicina $50 \mathrm{mg} / \mathrm{m}^{2}$ e ciclofosfamida 500 $\mathrm{mg} / \mathrm{m}^{2}$ ) e FEC 100 no grupo B (5-FU $500 \mathrm{mg} / \mathrm{m}^{2}$, epirrubicina $100 \mathrm{mg} / \mathrm{m}^{2}$ e ciclofosfamida $500 \mathrm{mg} / \mathrm{m}^{2}$ ).

Foram analisados, durante a quimioterapia, o estado geral, variação ponderal, alopécia, alterações digestivas, hematológicas e cardiotoxicidade. Após a quimioterapia avaliou-se a resposta tumoral clínica e, na peça cirúrgica, a resposta anatomopatológica.

O estado geral alterou-se em 22 pacientes (36\%), sendo que 5 (8\%) no grupo A e 17 (28\%) no grupo B. A maioria, 38 pacientes (64\%), manteve seu estado geral inicial inalterado. Verificou-se um maior ganho ponderal no grupo $\mathrm{B}$, porém dentro de valores médios.

Evidenciou-se uma diminuição progressiva dos valores hematológicos médios. No grupo A não houve necessidade de redução ou adiamento das doses. No grupo B a mielossupressão foi mais intensa e determinou redução das doses em 34 ciclos (28\%) de 16 pacientes, porém não levou a situações clínicas de risco de vida.

Náuseas e vômitos ocorreram em 68,7\% dos 
ciclos. No grupo B houve um maior percentual de formas severas. A diarréia foi manifestação digestiva pouco freqüente, comprometendo apenas 6 ciclos e sempre de forma leve.

Houve redução nos niveis da fração de ejeção ventricular esquerda (FEVE) em 90\% dos casos, sendo $80 \%$ no grupo A e $100 \%$ no grupo B. Em apenas 8 casos (13\%) a FEVE esteve abaixo do normal, sendo 2 (6\%) no grupo A e 6 (20\%) no grupo B. Nas outras pacientes as reduções da FEVE foram menores que 20\%. Houve alterações inespecíficas no eletrocardiograma (ECG). As mais freqüentes foram alterações da repolarização ventricular, taquicardia e extra-sístoles. As alterações da FEVE e ECG foram mais freqüentes e intensas no grupo B, porém não tiveram manifestações clínicas.

A resposta clínica objetiva (resposta completa e resposta parcial) nos grupos A e B foi 93\% e 96\%, respectivamente. Os resultados foram similares, porém no grupo B houve um percentual maior de respostas clínicas completas.

Avaliou-se a doença residual no sítio primário e nos linfonodos axilares. No grupo A houve 4 (13\%) casos de reposta anatomopatológica completa, 12 (40\%) de tumor residual microscópico e 14 (47\%) de tumor macroscópico. No grupo B ocorreram 10 (33\%) casos de resposta anatomopatológica completa, 7 (23\%) de tumor residual microscópico e 13 (44\%) de tumor macroscópico.

Concluiu-se que a quimioterapia primária proporcionou resposta clínica e anatomopatológica maior no grupo com intensificação de dose. A toxicidade, tolerável e reversível, foi mais acentuada no grupo de altas doses.

Palavras-chave: Mama: câncer. Quimioterapia. Quimioterapia neoadjuvante.

\section{Influência da Tibolona e do Exercício Físico Aeróbio sobre a Antropometria e o Perfil Lipídico na Menopausa}

Dissertação de Mestrado, apresentada ao Departamento de Ginecologia e Obstetrícia da Faculdade de Medicina de Botucatu da Universidade, Estadual Paulista, em 14/11/97.

Autor: Eliana Aguiar Petri Nahás

Orientador: Prof. Dra. Anagloria Pontes

Na menopausa há aumento do depósito de gordura abdominal influenciado pelo hipoestrogenismo e hiperandrogenismo relativo. A obesidade androgênica é metabolicamente diferente da ginecóide, porque apresenta maiores taxas de lipólise, com conseqüentes alterações negativas no perfil lipídico e aumento do risco para doença cardiovascular (DCV). O objetivo deste estudo foi avaliar as repercussões sobre a antropometria e perfil lipídico da mulher em menopausa, submetidas ao exercício físico aeróbio e à tibolona. Dezenove mulheres menopausadas foram divididas em 2 grupos: obesas $(n=12)$ e não-obesas $(n=7)$ e seguidas por 18 meses. As pacientes realizaram exercício físico aeróbio isolado, por 6 meses, com duração de 75 minutos, em três períodos semanais. O exercício foi associado à tibolona, na dose oral de 2,5 mg/dia, durante 12 meses. A antropometria foi avaliada pelo índice de massa corpórea (IMC), pela relação cintura-quadril (RCQ) e pela medida de pregas cutâneas. Considerou-se obesidade o $\mathrm{IMC}^{3} 30 \mathrm{~kg} / \mathrm{m}^{2}$ e a distribuição de gordura androgênica, quando $\mathrm{RCQ}>0,80$. A porcentagem de gordura corporal, obtida pelo somatório das medidas de pregas cutâneas, foi considerada elevada, acima de 30\%. Avaliou-se no perfil lipídico o colesterol total, HDL, LDL, VLDL e triglicerídeos. Inicialmente, observou-se o colesterol total e LDL elevados, com HDL, VLDL e triglicerídeos normais em todas as pacientes. Independentemente do IMC e da porcentagem de gordura corporal, as pacientes apresentavam RCQ androgênica. Com exercício físico aeróbio isolado, ocorreu redução da porcentagem de gordura corporal e queda do colesterol total, LDL, VLDL e dos triglicerídeos, ao passo que o HDL, o IMC e a RCQ não se alteraram. Quando associado à tibolona não se observou ganho de peso ou de gordura corporal, mas ocorreu diminuição da RCQ. Os valores de colesterol total e LDL mantiveram-se inalterados, ao passo que houve queda acentuada do HDL com 6 meses da tibolona, retornando seus valores próximos aos basais no final dos 12 meses. Os triglicerídeos e VLDL continuaram em decréscimo significativo até o final do estudo. Observou-se homogeneidade das respostas entre obesas e não-obesas quanto ao perfil lipídico e parâmetros antropométricos. O exercício físico contribuiu para redução da porcentagem de gordura corporal e dos níveis do colesterol total e LDL e, a tibolona para diminuição da RCQ e dos triglicerídeos e VLDL. Estes resultados sugerem que o exercício físico aeróbio e a tibolona podem ter efeitos benéficos e complementares sobre a antropometria e o perfil lipídico, na mulher em menopausa.

Palavras-chave: Menopausa. Perfil lipídico. Exercício físico. Terapia de reposição hormonal. 